\title{
Progressive Hyperpigmentation in a Child: An Early Marker of Infantile Tremor Syndrome
}

\author{
Shivi Nijhawan ${ }^{1}$, Anviti Gupta ${ }^{1}$, Manisha Nijhawan ${ }^{1}$, Divya $\operatorname{Yadav}^{1}$, Madhu Mathur $^{2}$ \\ ${ }^{1}$ Department of Dermatology, Mahatma Gandhi Medical Hospital, Jaipur, India \\ ${ }^{2}$ Department of Paediatrics, Mahatma Gandhi Medical Hospital, Jaipur, India \\ Email address: \\ shivi.nijhawan@yahoo.com (S. Nijhawan), anvitigupta96@gmail.com (A. Gupta),m.nijhawan12@gmail.com (M. Nijhawan), \\ rockin.diva3@gmail.com (D. Yadav), madhumathur07@gmail.com (M. Mathur) \\ ${ }^{*}$ Corresponding author
}

\section{To cite this article:}

Shivi Nijhawan, Anviti Gupta, Manisha Nijhawan, Divya Yadav, Madhu Mathur. Progressive Hyperpigmentation in a Child: An Early Marker of Infantile Tremor Syndrome. American Journal of Pediatrics. Vol. 7, No. 3, 2021, pp. 170-173. doi: 10.11648/j.ajp.20210703.25

Received: July 13, 2021; Accepted: August 2, 2021; Published: September 4, 2021

\begin{abstract}
Hyperpigmentary disorders are commonly encountered group of dermatoses in the paediatric age group. One of the most common causes of progressive generalised hyperpigmentation in paediatric population is nutritional vitamin B12 deficiency (NVBD). NVBD can present with a bunch of signs and symptoms like skin hyperpigmentation, pallor and regression of neurodevelopment. We report a case of a plump looking 13 months old, girl child from non-consanguineous marriage of low socioeconomic family who presented with generalised hyperpigmentation, failure to thrive and regression of mile stones. Physical examination revealed anaemia and sparse, thin, lustreless hair with dull, apathetic look. A differential diagnosis of Addison's disease, hypothyroidism, and neurodegenerative disease, was considered based on clinical suspicion. She was further investigated by a paediatrician. Her blood investigations revealed low levels of Vitamin B12 (159 pg/dl) and haemoglobin (2.6 $\mathrm{g} / \mathrm{dl}$ ). On radiological examination, NCCT brain showed cortical atrophy. A final diagnosis of Infantile Tremor Syndrome (ITS) was made. ITS is a rare condition which presents with cutaneous manifestation like hyperpigmentation, scanty scalp hair along with lethargy, apathy along with signs and symptoms of neurodegeneration. Progressive cutaneous hyperpigmentation in a child can present as early marker of a rare neurodegenerative condition like ITS.
\end{abstract}

Keywords: Infantile Tremor Syndrome, Neurodegenerative Disease, Addison's Disease, Hypothyroidism, Inborn Error of Metabolism

\section{Introduction}

Hyperpigmentation can affect children, stemming from a large number of causes. Cutaneous hyperpigmentation can be classified as congenital or acquired. Congenital hyperpigmentation is usually related to gene changes and acquired is due to skin inflammation, systemic diseases, environmental factors or medications, among which the most common factors are metabolic causes and medications. Metabolic causes of hyperpigmentation include vitamin deficiencies, such as vitamin B12 and folic acid.

Infantile tremor syndrome is often associated with vitamin B12 deficiency. It is characterized by a tetrad of tremors, pallor, hyperpigmentation of skin, sparse brown hair and developmental regression. [1] It has been reported in children between 5 months and 3 years of age with a male preponderance [2]. In India, it accounts for 0.2 to $2 \%$ of paediatric hospital admissions $(1-2 \%$ in $1960 \mathrm{~s}, 1.1 \%$ in 1975-77 and 0.2\% in mid-1990s). [3] Better weaning practices as well as improvement in nutritional and living status could explain the reducing incidence rate over years. Most of the infants with ITS comes from low socioeconomic families. Vitamin B12 deficiency is considered as the diagnostic hallmark of ITS, though other nutritional deficiencies like Zinc, Magnesium have been found to be associated with ITS. It is commonly found in children who are exclusively breast fed by strict vegetarian mothers who are malnourished themselves, resulting from poverty and ignorance. The affected child presents with pallor, skin and hair changes, dull and apathic look due to compromised nutritional status. They usually have a plum body due to preservation of subcutaneous fat. 
Involuntary movements in the form of tremors supervene in the natural course of the illness in a significant number of cases. The children show regression of motor and cognitive milestones in around one year of age.

Even when vitamin B12 deficiency is quite common, infants are rarely brought to medical attention because of skin hyperpigmentation. Natural dark complexion of Indian infants also makes it difficult for parents to notice gradual changes in the skin color. This was in contrast to our case, where the child was taken to a local hospital for weakness and lethargy, and after a few months was presented in dermatology opd for progressive darkening of the skin color.

We, hereby report a case of a 13-month-old baby who was presented in our dermatology opd for the complaints of gradual, progressive hyperpigmentation, along with sparse and brittle hair and apathic look. On further questioning the parents, a history suggestive of slowing of neurological development was revealed.

\section{Case Report}

A 13 months old girl child, born at term by LSCS to non-consanguineous parents of low socioeconomic status came to the skin OPD with the chief complains of increased darkening of skin for 3 months of age, decreased activity and loss of previously acquired milestones from past 2-3 months.

Hyperpigmentation of skin began at 3 months of age, initially involving extremities (palms \& soles), finger\& toes which eventually progressed to buttocks, back \& chest, lips \& palate. Her parents were concerned about her losing the previously acquired milestones. She was unable to stand and sit without support from last 3 months. Her parents noticed she was dull, lethargic and showed decreased activity from about the same time. She was taken to a local hospital for these complains and was given a few medications but did not show any improvement. She was predominantly breast fed by vegetarian mother and was partially immunized. There was no past history of any recurrent infections, any major illness, seizures, abnormal body movement, trauma nor there was any history of similar complaints in other members of the family.

On general physical appearance of the child, she had revealed she was lethargic, dull, plump, pale with apathetic look. Her weight was $6.9 \mathrm{~kg}$ and height were $69 \mathrm{~cm}$, both being below $3^{\text {rd }}$ percentile depicting moderate acute malnutrition. On cutaneous examination there was knuckle pigmentation [figure 2], generalized hyperpigmentation [figure1] (predominantly over palmar crease [figure 3]; sparing face, oral cavity and genetalia) and sparse, dry, lusterless hair.

On central nervous system evaluation, child was found to be irritable, dull, with decreased activity but power, tone and reflexes in bilateral Upper and Lower extremities were normal. Respiratory and cardiovascular examination was normal.

Based on history and clinical examination a differential diagnosis of Addison's disease, hypothyroidism, and neurodegenerative disease, were considered. She was referred to the pediatrics department for further evaluation.
Investigations done showed dimorphic anaemia with peripheral blood showing both microcytic as well as macrocytic RBCs. Further workup done for anaemia showed low levels of Vitamin B12 (159 pg/dl) and haemoglobin (2.6). Radiological examination, NCCT brain exhibited cortical atrophy. Other special investigations like Serum Cortisol, Serum ACTH, thyroid hormone, ABG and Fundus examination revealed normal results.

A final diagnosis of Infantile Tremor Syndrome (ITS) was made. The child received packed red blood cells (100 ml, Inj. B 12 (1000 microgram on alternate day), syrup Multivitamin, syrup Iron ( $3 \mathrm{ml} \mathrm{od}$ ) and syrup Calcium and vitamin D along with initiation of complementary feeding showing improvement. Within 48-72 hours there was improvement in activity and responsiveness. Hematological recovery was observed within 5-7 days. Skin pigmentation started to resolve in about 2-4 weeks. Lost developmental milestones began to return by 4 weeks and hair changes took several months.

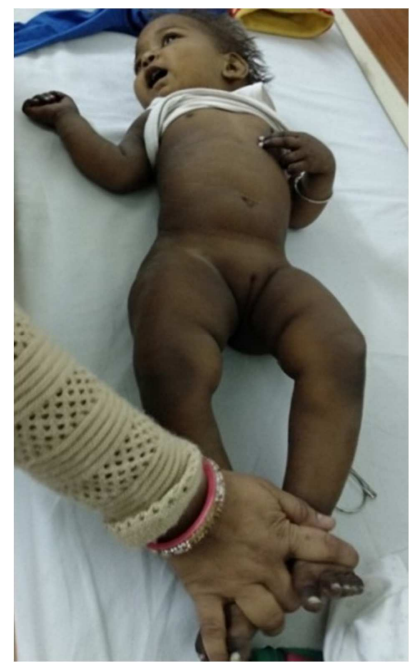

Figure 1. Generalized diffuse hyperpigmentation

\section{Discussion}

Progressive diffuse hyperpigmentation in a child can be a presentation of various underlying conditions. Some of the common associated conditions are- endocrinal disorders like Addison's disease, pheochromocytoma, Cushing's syndrome, thyroid disorders; nutritional deficiencies such as Kwashiorkar, folic acid deficiency, vitamin B12 deficiency; metabolic conditions like Porphyria cutanea tarda (PCT), hemochromatosis, wilson's disease, chronic renal failure (CRF); medications like minocycline, bleomycin, zidovudine, etc; autoimmune conditions like systemic sclerosis; miscellaneous conditions like baby syndrome and familial progressive hyperpigmentation. One of the most common cause of progressive generalized hyperpigmentation in Indian pediatric population is nutritional vitamin B12 deficiency (NVBD). 


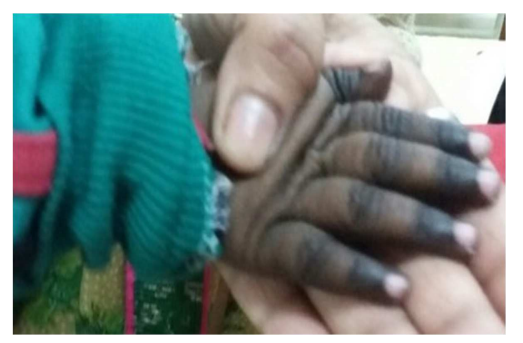

Figure 2. Knuckle pigmentation.

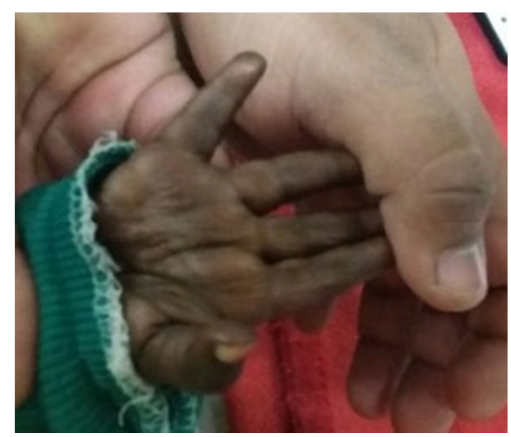

Figure 3. Hyperpigmentation accentuated over palmer creases.

In infants and young children, vitamin B12 deficiency is manifested always secondary to maternal vitamin B12 deficiency and is, therefore, seen in exclusively breastfed infants of mothers who are either strict vegetarian or have an underlying pernicious anemia. [4-6]. NVBD can affect hematologic, gastrointestinal and nervous system. [7] Infant with vitamin B12 deficiency clinically presents as apathy, neurologic symptoms of irritability, psychomotor regression, hypotonia, and involuntary movements. [4-6, 8] Muco-cutaneous involvement occurs as glossitis, angular cheilitis, skin hyperpigmentation, and hair abnormalities. [4-6]. Hair changes have been reported in 50\%-100\% Indian infants with vitamin B12 deficiency, as in our case. Almost all the children with associated vitamin B12 deficiency present with lusterless, sparse and brown hair.

Skin hyperpigmentation has been reported to be uncommon in Caucasian infants with vitamin B12 deficiency $[9,10]$ but cutaneous manifestations are consistently present in Indian [4, 11] infants with vitamin B12 deficiency.

Pathophysiology of hyperpigmentation is still debatable. According to a hypothesis proposed by Gilliam and Cox, patients with vitamin B12 deficiency have low levels of reduced glutathione (GSH). It is known that GSH inhibits tyrosinase activity and consequently melanogenesis. Decreased levels of GSH allow increased tyrosinase activity, and thus favoring increased melanogenesis [12]. The mechanism of hyperpigmentation is due to increased melanin synthesis rather than any defect in melanin. Characteristic vitamin B12 hyperpigmentation includes hyperpigmentation of the extremities - especially over the dorsum of the hands and feet, with accentuation over the interphalangeal joints and terminal phalanges - associated with pigmentation of oral mucosa [13]. Morphologically, hyperpigmentation is observed as homogeneous or uniform darkening of skin [14, 15] or more commonly, as a network of light central areas and darker periphery, producing a peculiar reticulate or "honeycomb" pattern. This latter type of pigmentation has been reported only in Indian infants with vitamin B12 deficiency [15-20]. Mostly it is the dorsa of hands and feet which shows the most pronounced pigmentation, with accentuation over the interphalangeal joints, the so called "knuckle pigmentation." [4, 14, 12, 21] Rarely skin hyperpigmentation is generalized. [4, 14, 15] Our case presented with hyperpigmentation of skin, initially involving extremities (palms \& soles), finger\& toes which eventually progressed to buttocks, back \& chest, lips \& palate. Since tremors is the usual presentation, the term "infantile tremor syndrome" has been used in the Indian literature to describe this disorder. [4, 8, 15-22]. In our case however, there was no history of abnormal body movements, seizures or coarse tremors. ITS is a self-limiting disorder commonly observed between 5 months to 3 years of age and is clinically characterized by anemia, skin pigmentation, coarse tremors and mental regression in a plump looking child. Recently, Vitamin B12 has been shown to be strongly associated with ITS [23] and it has been suggested to rename it as "nutritional vitamin B12 deficiency in infants". [23] This was mainly because of some similar cases in western world. An assessment of Iron, Vitamin B12 and Folate status in 50 children with ITS showed Vitamin B12 deficiency in 70\% children while none of them had Iron or Folate deficiency. [24] Vitamin B12 deficiency is targeted because mothers of ITS children are vegetarian and their complementary feeds are also delayed. $[15,21]$ Our case was also exclusively breast fed by a strict vegetarian mother. In the index case, a diagnosis of infantile tremor syndrome was considered based on pallor, developmental delay/regression, skin hyperpigmentation and brown scanty scalp hair. Regular monitoring of vitamin B12 levels in mothers if clinically suspected in last trimester and improving weaning practices can help in the prevention of ITS in the first place. Early recognition and prompt treatment of this condition are extremely important to prevent the deleterious effects of vitamin B12 deficiency on the growing brain. [4, 5] Management of ITS being largely empirical, it includes vitamin B12, folic acid, calcium, zinc, iron, magnesium and high protein diet.

\section{Conclusion}

ITS should be considered in any child $<3$ years presenting with skin hyperpigmentation and sparse hair, anemia, developmental delay/regression, with or without tremors. Progressive cutaneous hyperpigmentation in a child can present as an early marker of a neurodegenerative condition like ITS. Therefore, ITS should be excluded in any child presenting with neuroregression and mucocutaneous signs of vitamin B12 deficiency. Such cases of ITS are very common in pediatric opd but in an individual presenting with diffuse hyperpigmentation to a dermatologist, they need to develop an insight to consider neurodegenerative or metabolic disorders also.

Since vitamin B12 deficiency is quite common in India due 
majority of vegetarian population, any patient of pediatric age group presenting with symptoms of generalised hyperpigmentation, failure to thrive, anemia and regression of mile stones should be worked up thoroughly.

\section{References}

[1] Dikshit AK. Nutritional dystrophy with anaemia. Indian J Child Health. 1957; 6: 132-6.

[2] Gupte S, Pal M, Gupta SK, Sangra KR. Infantile tremor syndrome [ITS] In: Gupte S, Editor. Textbook of paediatric nutrition. New Delhi: Peepee; 2006: 255-265.

[3] Gupte S. Infantile tremor syndrome. In: Gupte S, ed. The Short Textbook of Paediatrics, 10th ed. New Delhi; Jaypee Brothers. 2004: 716-9.

[4] Goraya JS, Kaur S, Mehra B. Neurology of nutritional vitamin B12 deficiency in infants. Case series from India and literature review. J Child Neurol. 2015; 30: 1831-1837.

[5] Korenke GC, Hunneman DH, Eber S, Hanefeld F. Severe encephalopathy with epilepsy in an infant caused by subclinical maternal pernicious anemia: case report and review of the literature. Eur J Pediatr. 2004; 163: 196-201a.

[6] Incecik F, Herguner MO, Altunbasak S, Leblebisatan G. Neurological findings of nutritional vitamin B12 deficiency in children. Turk J Pediatr. 2010; 52: 17-21.

[7] Oh R, Brown DL. Vitamin B12 deficiency. Am Fam Physician 2003; 67 (5): 979-86.

[8] Jadhav M, Webb JKG, Vaishnava S, Baker SJ. Vitamin B12 deficiency in Indian infants. Lancet. 1962; 1: 903-990.

[9] Johnson PR, Roloff JS. Vitamin B12 deficiency in an infant strictly breast-fed by a mother with latent pernicious anemia. J Pediatr. 1982; 100: 917-919.

[10] Higginbottom MC, Sweetman L, Nyhan WL. A syndrome of methylmalonic aciduria, homocystinuria, megaloblastic anemia and neurologic abnormalities in a vitamin B12-deficient breast-fed infant of a strict vegetarian. N Engl J Med. 1978; 299: 317-323.

[11] Sharda B, Bhandari B. Infantile tremor syndrome. Indian Pediatr. 1987; 24: 415-421.
[12] Gilliam JN, Cox AJ. Epidermal changes in vitamin B12 deficiency. Arch Dermatol. 1973; 107: 231-6.

[13] Aaron S, Kumar S, Vijayan J, Jacob J, Alexander M, Gnanamuthu C. Clinical and laboratory features and response to treatment in patients presenting with vitamin B12 deficiency-related neurological syndromes. Neurol India 2005; 53 (1): 55-8.

[14] Demir N, Dogan M, Koc A, et al. Dermatological findings of vitamin B12 deficiency and resolving time of these symptoms. Cutan Ocul Toxicol. 2014; 33: 70-73.

[15] Sachdev KK, Manchanda SS, Lal H. The syndrome of tremors, mental regression and anemia in infants and young children. A study of 102 cases. Indian Pediatr. 1965; 2: 239-251.

[16] Pohowalla JN, Kaul KK, Bandari NR, Singh SD. Infantile "meningo- encephalitic" syndrome. Indian J Pediatr. 1960; 27: $49-54$.

[17] Kaul KK, Prasan NG, Chowdhary RM. Further observations on the syndrome of tremors in infants. Indian Pediatr. 1964; 1: 219-225.

[18] Srikantia SG, Reddy V. Megaloblastic anemia of infancy and vitamin B12. Br J Hematol. 1967; 13: 949-953.

[19] Bajpai PC, Misra PK, Tandon PN. Further observations on infantile tremor syndrome. Indian Pediatr. 1968; 5: 297-307.

[20] Ramakumar L, Pandove SP. Infantile tremor syndrome. Indian J Pediatr. 1975; 42: 215-225.

[21] Baker SJ, Ignatius $\mathrm{M}$, Johnson $\mathrm{S}$, Vaish SK. Hyperpigmentation of skin. A sign of vitamin B12 deficiency. Br Med J. 1963; 1: 1713-1715.

[22] Garewal G, Narang A, Das KC. Infantile tremor syndrome: a vitamin B12 deficiency syndrome in infants. J Trop Pediatr. 1988; 34: 174-178.

[23] Goraya JS, Kaur S. Infantile tremor syndrome - downbut not out. Indian Pediatr. 2015; 52: 249-250.

[24] Rajpoot KS, Poswal L, Goyal S. Assessment of iron, folate and vitamin B12 status in children with infantile tremor syndrome. Int J Contemp Pediatr. 2016; 3: 587-592. 